\title{
Preventive Health Behavior and Adaptive Accuracy of Risk Perceptions
}

\author{
Britta Renner, ${ }^{1 *}$ Benjamin Schüz, ${ }^{2}$ and Falko F. Sniehotta ${ }^{3}$
}

\begin{abstract}
This study examined the relation between health behavior and risk perceptions in the context of an acute livestock epidemic. Participants in a longitudinal web-based survey $(N=195)$ were asked to report their meat consumption and their perceived risk in relation to bovine spongiform encephalopathy (BSE) and other related livestock diseases. Cross-sectional analyses at both measurement points ( $\mathrm{T} 1$ and $\mathrm{T} 2$ ) showed that participants with low levels of preventive nutrition (high meat consumption) felt more at risk for BSE-related diseases than those reporting comparable higher levels of preventive behavior (low meat consumption), indicating relative accuracy. These results suggest that people recognize when their behavior is risky. More importantly, perceived risk also showed adaptive accuracy from a change perspective: increases in preventive nutrition from $\mathrm{T} 1$ to $\mathrm{T} 2$ were significantly associated with decreases in perceived risk between T1 and T2. Possible foundations and implications of an adaptive accuracy of risk perceptions are discussed.
\end{abstract}

KEY WORDS: Accuracy; bovine spongiform encephalopathy (BSE); health behavior change; risk perception

\section{INTRODUCTION}

The idea that people adopt precautious health behaviors in order to reduce risks for their health is explicitly or implicitly inherent in most social cognition theories of health behavior (Weinstein et al., 1998). Accordingly, health promotion and health education programs often provide people with risk information in order to motivate them to take precautious actions. Thus, most research on the risk perceptionpreventive behavior relation has consequently focused on the question whether risk perceptions

\footnotetext{
1 Department of Psychology, University of Konstanz, Germany.

2 Jacobs Center on Lifelong Learning and Institutional Development, Jacobs University Bremen, Germany.

3 School of Psychology, University of Aberdeen and Aberdeen Collaboration on Research in Nutrition, Aberdeen, UK.

* Address correspondence to Britta Renner, Department of Psychology, Psychological Assessment, University of Konstanz, PO Box D 47, 78457 Konstanz, Germany; britta.renner@unikonstanz.de.
}

motivate subsequent behavior changes (for metaanalyses, see Gerrard et al., 1996; Harrison et al., 1992; Sheeran \& Abraham, 1996).

\subsection{Adaptive Accuracy in Risk Perceptions}

Assuming that people take precautions in order to reduce their risk implies that they will perceive themselves as being less at risk as a consequence of their behavior change (Brewer, Weinstein, Cuite, \& Herrington, 2004; Weinstein, 2003). Thus, if people have taken precautions in order to reduce their personal risk, they should subsequently show adaptive decreases in risk perceptions. Empirically, this would result in a positive correlation between risk perceptions at an initial point in time (T1) and preventive behavior at a subsequent point in time (T2), and a negative correlation between behavior change and risk perceptions at the subsequent point in time (T2) (Weinstein et al., 1998). Both from a theoretical and a practical view this adaptive accuracy of risk 
perception is of crucial importance: If people change behaviors and decrease their risk perceptions as a consequence, they will be more motivated to maintain the new behavior pattern. However, if people still feel at risk despite having changed their behavior, they will be less likely motivated to maintain the behavior change in the long run and this may even lead to behavior disengagement as a consequence.

While there is a wealth of empirical studies examining the impact of risk perceptions on subsequent behavior changes, the aspect of adaptive accuracy of risk perception has been comparably neglected so far. Studying the adaptive accuracy of risk perceptions requires a longitudinal research design with risk perceptions and behavior assessed at an initial measurement point (T1) and then reassessed at a later date (T2). Brewer et al. (2004) reported first evidence for adaptive accuracy in risk perception in the context of a novel preventive health behavior-lyme disease vaccination. They found that participants who had been vaccinated showed, at a second measurement point, a greater decrease in their risk perceptions than participants who were still unvaccinated. This suggests that adopting preventive behavior leads to an adaptive reappraisal of personal risk. It is important to note that, as Brewer et al. (2004) argued, adaptive responses in risk perception do by no means imply that people think that preventive behavior entirely eliminates the risk.

Assuming that adaptive accuracy manifests in changes in risk perceptions suggests that people who have adopted precautious behavior changes should show a greater decrease in risk perceptions than those who have not. However, despite having adopted preventive behaviors, which led to lower risk perceptions, people starting with high risk perceptions at the beginning might still feel more at risk than people who have not changed their behavior. Thus, adaptive accuracy manifests in absolute changes within the individual, but not necessarily in rank order changes between individuals. This might complicate the interpretation of risk perception-behavior relations: from a longitudinal perspective, individuals may show adaptive accuracy (their risk perceptions have decreased) but from a cross-sectional perspective they may show relative inaccuracy because people engaging in preventive behaviors might have, nevertheless, higher risk perceptions at that time point than people not having adopted precautions. For example, in order to reduce their elevated perceptions of risk for diseases associated with the consumption of potentially contaminated food, some people might refrain from eating certain foods, which in turn leads them to lower their risk perceptions. Other people might perceive themselves not at risk right from the beginning and thus might not change their behavior and maintain low levels of risk perception. Hence, adaptive accuracy of risk perceptions in longitudinal designs is silent in terms of relative accuracy in cross-sectional designs and vice versa.

\subsection{Minimization of Risk Perceptions}

According to prevailing theories of health behavior change (Armitage \& Conner, 2000; Renner \& Schwarzer, 2003; Rutter \& Quine, 2002), a decrease in risk perception over time is conceptualized as an adaptive consequence of behavior change (Brewer et al., 2004). Conversely, from a social cognitive perspective, a decrease in risk perception could also represent a biased reaction caused by repeated exposure to high risk. Research on cognitive biases has shown that repeated exposure to hazards leads to an increase in confidence and perceptions of personal control (Johnson \& Tversky, 1983; Slovic \& Lichtenstein, 1982). In a similar vein, empirical studies in the context of technological hazards have also shown evidence for such habituation effects. In a longitudinal study, Lima (2004), for example, observed that people living close to an incinerator showed a decrease in risk perceptions after a five-year exposure (see also Weyman \& Clarke, 2003). For risks emerging from individual behavior, Brown (2005) accordingly argued that repeated risk-taking behavior (in this case speeding) might lead to reappraisals of the dangers inherent in behavior if risk takers do not experience negative consequences. This tendency to perceive a hazard less threatening and risky as a function of repeated exposure might be facilitated through the desire to maintain or regain a positive view of the self and feelings of control (Brown, 2005; Croyle et al., 1997; Gerrard et al., 2000; Kunda, 1990). Thus, from a social cognitive perspective, people might reduce their risk perceptions although they have not changed their risk behavior. In line with this minimization hypothesis, Brown (2005) showed that high-risk-taking behavior at an initial measurement point in time (T1) was associated with low risk perceptions at a subsequent measurement point in time (T2).

Thus, when examining the relations between behavior and risk perceptions, both positive and negative relations can be expected. According to theories of health behavior, the adoption of precautionary behavior is followed by a decrease in risk perceptions 
(adaptive accuracy hypothesis), resulting in a negative cross-lagged relation (cf. Brewer et al., 2004; Weinstein et al., 1998). On the other hand, from a social-cognition perspective, repeated performance of risk behavior should lead to a decrease in risk perception over time due to increased feelings of control and self-defensive strivings (minimization hypothesis), thus yielding a positive cross-lagged relation. From a methodological point of view, cross-sectional correlations are only informative in terms of relative accuracy in risk perceptions. For testing the adaptive accuracy and minimization effects, cross-lagged correlations with changes in behavior and risk perceptions need to be investigated (cf. Weinstein, 2007).

\subsubsection{The Present Study}

This study aims at examining the relation between health behavior and risk perceptions. According to Weinstein et al. (1998), the occurrence of new hazards or new possibilities for precaution is destabilizing and prompts people to consider changing their behavior. The coinciding outbreaks of BSE (Bovine Spongiform Encephalopathy, "mad cow disease") and FMD (Foot and Mouth Disease) epidemics in Germany in early 2001 were newly emerging hazards at the time of data collection and were thus selected as context for the study (Sniehotta et al., 2005). Both BSE and FMD posed different potential threats for health, as transmission was unclear at the time (Pfister \& Böhm, 2001). Refraining from or reducing red meat consumption was therefore viewed as appropriate precautious behavior at that time. The two measurement points of the study (between April 2001 and August 2001) were chosen in close temporal coincidence following the outbreak of BSE and FMD in Germany in November 2000 and public discussions about new legislation (February 2001).

In particular, the following three hypotheses were tested. (1) Relative accuracy: From a cross-sectional perspective, it is expected that people show relative accuracy in risk perceptions, indicated by a negative cross-sectional correlation between risk perception and preventive health behavior at an initial measurement point in time (T1) and at a subsequent measurement point in time (T2), respectively. (2) Adaptive accuracy: From a longitudinal perspective, it is predicted that adopting precautious behavior between $\mathrm{T} 1$ and $\mathrm{T} 2$ is accompanied by a decrease in risk perception between T1 and T2. (3) Minimization hypothesis: High levels of risk behavior at $\mathrm{T} 1$ are associated with a decrease in risk perception between $\mathrm{T} 1$ and $\mathrm{T} 2$.

\section{METHOD}

\subsection{Sample and Procedure}

In a longitudinal online survey in close temporal coincidence to the BSE and FMD epidemics in Germany in 2001, 195 participants completed htmlquestionnaires assessing sociodemographic variables (age, gender, and education), meat consumption, and risk perceptions at two time points (T1 and $\mathrm{T} 2$ ) with a time lag of four months. After excluding 48 participants who indicated being vegetarians, 147 participants remained for analyses. Vegetarians were excluded because by refraining from meat they already follow preventive measures. The final study sample comprised 81 women and 66 men with an age range between 16 and $64(M=31.16 ; S D=9.77$ years). Education of the sample was high: $83.7 \%$ of the sample indicated A-levels ("Abitur"), 13.6\% reported lower qualifications.

\subsection{Measures}

Risk perceptions were assessed with two items adapted from Weinstein (2003) at T1 $(r=0.81, p<$ $0.001)$ and at T2 $(r=0.76, p<0.001)$, namely: "Compared to other persons of my age and gender, my risk of contracting (a) Creutzfeldt-Jakob disease (new Variant)..., (b) another animal-related disease...". Items were answered on a seven-point Likert scale from "much less than average" (1) to "much more than average" (7).

Meat consumption was assessed at T1 $(r=0.59$, $p<0.001)$ and at T2 $(r=0.67, p<0.001)$ using ratings of consumption frequency of red meat and beef on sixpoint scales ranging from "never" (1), "less than once a week" (2), "1-2 times a week" (3), "several times a week" (4), "daily" (5), and "several times daily" (6). The items were: "How often do you eat the following products?" followed by (a) "beef or products with beef" and (b) "red meat or red meat products." Scale means, $S D$, range, and intercorrelations are reported in Table I.

\subsection{Analytic Procedure}

After imputing missing values, ${ }^{4}$ mean scores of risk perception and preventive nutrition were computed. The items assessing meat consumption were reversely coded and transformed into a measure of

4 As missing values on no variable exceeded 5\%, estimation maximization imputation (Enders, 2001) in SPSS 12 was used to impute missing values. 


\begin{tabular}{lcccccc}
\hline Variable & 2 & 3 & 4 & $M$ & $S D$ & $r^{a}$ \\
\hline 1. Risk perception T1 & $0.64^{* * *}$ & $-0.52^{* * *}$ & $-0.58^{* * *}$ & 3.23 & 1.07 & $0.81^{* * *}$ \\
2. Risk perception T2 & & $-0.44^{* * *}$ & $-0.54^{* * *}$ & 3.30 & 0.99 & $0.76^{* * *}$ \\
3. Preventive nutrition T1 & & & $0.78^{* * *}$ & 5.38 & 0.89 & $0.59^{* * *}$ \\
4. Preventive nutrition T2 & & & & 5.32 & 0.83 & $0.67^{* * *}$ \\
\hline
\end{tabular}

${ }^{\mathrm{a}}$ Interitem-correlation.

*** $p<0.001$.

Response range between 1 (far below average) to 7 (much more than average) for risk

perceptions; 6 (never beef or meat) to 1 (meat or beef several times daily) for preventive nutrition.
Table I. Intercorrelations, Means, and $S D$ Between Scales and Interitem Correlations for Each Scale preventive nutrition, so that lower levels of beef and red meat consumption indicate higher levels of preventive nutrition. An index of behavior change was obtained by subtracting $\mathrm{T} 1$ preventive nutrition from $\mathrm{T} 2$ preventive nutrition. The hypotheses were tested by means of path analysis allowing covariances between the predictor variables to be freely estimated, controlling for the interrelations of risk perceptions $\mathrm{T} 1$, preventive nutrition $\mathrm{T} 1$, and changes in preventive nutrition from $\mathrm{T} 1$ to $\mathrm{T} 2$.

\section{RESULTS}

The cross-sectional perspective shows substantial negative correlations between risk perceptions and preventive nutrition. Participants who reported less preventive behaviors, that is, consumed more meat and beef, felt more at risk for BSE or other livestock diseases than participants who reported more preventive behaviors, that is, consumed less beef and other red meat. The relationship was in the high effect size range at $\mathrm{T} 1(r=-0.52, p<0.001)$ and at $\mathrm{T} 2(r=-0.54$, $p<0.001)$. This negative cross-sectional relationship between risk perception and preventive behavior indicates relative accuracy at both measurement points.

In a second step, a longitudinal perspective was taken in order to analyze changes in risk perceptions in relation to behavior change.

Fig. 1 shows the results of the path analysis with $\mathrm{T} 1$ to $\mathrm{T} 2$ changes in risk perceptions as the dependent variable and risk perceptions at T1, preventive nutrition at $\mathrm{T} 1$, and changes in preventive nutrition as predictor variables.

Correlations between the independent variables show that high risk perceptions at $\mathrm{T} 1$ were negatively associated with preventive nutrition at $\mathrm{T} 1$, reflecting relative accuracy $(r=-0.52, p<0.001)$. Moreover, a low rate of preventive nutrition at $\mathrm{T} 1$ was related to greater positive changes of preventive nutrition behavior between T1 and T2 $(r=-0.31, p<$ 0.001 ). The relation between risk perception at T1 and changes in preventive nutrition was statistically not significant.

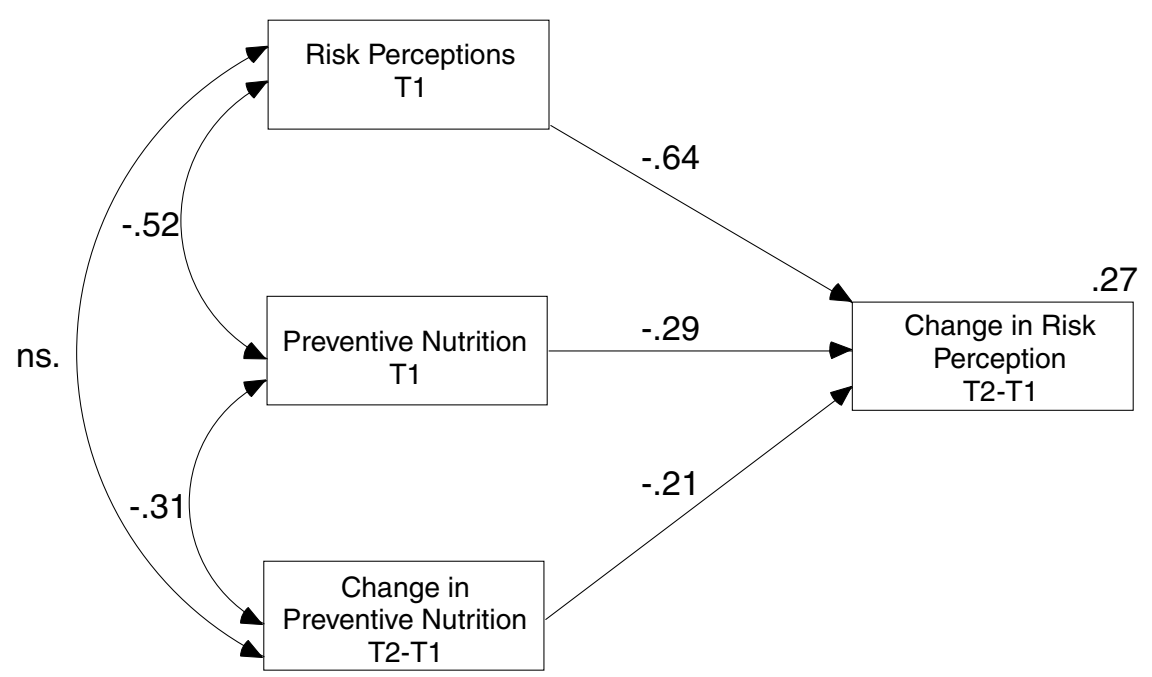

Note. Preventive nutrition has been reversely coded; higher levels of preventive nutrition indicate lower levels of meat consumption. All coefficients are significant at $p<0.05$.
Fig. 1. Path analysis (standardized solution). 
Risk perception at $\mathrm{T} 1$ was significantly related to changes in risk perceptions between $\mathrm{T} 1$ and $\mathrm{T} 2$ (beta $=-0.64, p<0.001$ ), indicating that high risk perceptions at $\mathrm{T} 1$ were associated with a decrease in risk perception between T1 and T2. Moreover, a negative standardized regression coefficient for the direct effect of preventive nutrition at $\mathrm{T} 1$ on changes in risk perceptions between $\mathrm{T} 1$ and $\mathrm{T} 2$ was found (beta $=-0.29, p<0.001)$. Hence, a higher rate of preventive nutrition at $\mathrm{T} 1$ was associated with a decrease in risk perceptions between T1 and T2. Finally, changes in preventive nutrition behavior between $\mathrm{T} 1$ and T2 were negatively associated with changes in risk perception between $\mathrm{T} 1$ and $\mathrm{T} 2$ (beta $=-0.21, p$ $<0.01$ ). Thus, positive changes in preventive nutrition between $\mathrm{T} 1$ and $\mathrm{T} 2$ were related to a decrease in risk perceptions between $\mathrm{T} 1$ and $\mathrm{T} 2$. All three predictors, risk perceptions at $\mathrm{T} 1$, preventive nutrition at $\mathrm{T} 1$, and change in preventive nutrition explained $27 \%$ of variance in the risk perception change score.

In order to underpin the results, an alternative path model including risk perception at $\mathrm{T} 2$ as the dependent variable was additionally calculated. The analysis revealed a positive relation between risk perceptions at $\mathrm{T} 1$ and risk perceptions at $\mathrm{T} 2$ (beta $=0.49$, $p<0.001)$, indicating stability in risk perception over time. In addition, higher preventive nutrition at $\mathrm{T} 1$ was related to lower risk perception at $\mathrm{T} 2$ (beta $=$ $-0.25, p<0.001)$. Finally, adopting preventive nutrition behavior between $\mathrm{T} 1$ and $\mathrm{T} 2$ was associated with lower risk perception at T2 $($ beta $=0.18, p<0.01)$. All three predictors explained $47 \%$ of the total variance in risk perception at $\mathrm{T} 2$.

\section{DISCUSSION}

The relation between risk perceptions and health behavior is a core aspect of current health behavior theories (Renner \& Schwarzer, 2003; Weinstein, 2003). It is commonly assumed that perceptions of risk encourage people to take precautious action in order to reduce this risk (Brewer et al., 2004). As this motivation hypothesis (Weinstein et al., 1998) implies that a lack of preventive behavior is at least partly attributable to inaccurate risk perceptions, it is vital to examine the factors that influence the accuracy of risk perceptions. Accuracy of risk perceptions can be assessed in various ways. In this study, the accuracy of risk perceptions was examined both from a crosssectional and a longitudinal perspective. The crosssectional perspective allows for examining whether risk perceptions reflect concurrent risk-relevant behavior, thus indicating relative accuracy. The longitudinal perspective allows examining whether behavior changes covary with changes in perceived risk (adaptive accuracy).

\subsection{The Cross-Sectional View: Relative Accuracy of Risk Perceptions}

In accordance with current theoretical conceptions in risk perception research, these data revealed clear support for the relative accuracy hypothesis (cf. Weinstein et al., 1998) at both measurement points. Accordingly, participants with low levels of preventive nutrition (i.e., consuming more red meat during a livestock epidemic) felt more at risk than those with comparable higher levels of preventive behavior (i.e., eating less or no red meat). The finding of a substantial negative correlation between preventive nutrition and risk perception at both measurement points suggests that participants on average had accurate conceptions about their actual risk status. However, the correlation reveals only their relative standing on the risk behavior ladder and does not rule out that people may have misconceptions about the magnitude of their absolute risk or their risk in comparison to a reference group (cf. French \& Marteau, 2007; Weinstein, 2003).

It is important to note that the negative correlation between risk perceptions and preventive nutrition at both measurement points reflects accuracy in risk perceptions rather than an adverse motivational effect of high risk perceptions on preventive nutrition (van der Pligt, 1998; Weinstein \& Nicolich, 1993; Weinstein et al., 1998). Thus, interpreting crosssectional relations between risk perceptions and precautionary behavior in terms of the motivating effects of risk perceptions is inappropriate (Weinstein, 2007). If cross-sectional correlations are interpreted as indicators of the motivational effect of risk perception on health behavior, the observed negative relation between risk perception and preventive nutrition in this study would suggest that higher levels of perceived risk actually prevented participants from engaging in preventive nutrition. Or, on the other hand, that low levels of perceived risk encouraged preventive nutrition. Previous research has explained this contradictory finding by referring to defense or denial concepts (Ayanian \& Cleary, 1999; Sjöberg et al., 2004) or by arguing that risk perceptions are a consequence rather than an antecedent of engaging in preventive behavior (Reisen \& Poppen, 1999). However, cross-sectional relations between risk perceptions and 
preventive behaviors are silent in regard to the motivating effects of risk perceptions; they are merely descriptive: higher levels of risk perception covary with lower levels of preventive behavior, indicating that people realize when their behavior is risky (Weinstein, 2007).

\subsection{The Longitudinal View: Adaptive Accuracy of Risk Perceptions}

Assessing whether people engaging in risk behaviors are aware of their increased personal risk, however, is only one possibility to determine the accuracy of risk perceptions. If a preventive behavior is believed to reduce individual risk, according to the adaptive accuracy hypothesis people who take action should lower their personal risk perceptions (Brewer et al., 2004). This study accordingly found that increases in preventive nutrition from $\mathrm{T} 1$ to $\mathrm{T} 2$ were significantly associated with decreases in perceived risk between $\mathrm{T} 1$ and $\mathrm{T} 2$. This effect remained statistically significant even after controlling for correlations among baseline risk perception (T1) and baseline behavior (T1). Thus, preventive behavior changes appear to lead to corresponding changes in risk perception, indicating adaptive accuracy.

No supporting evidence was found for the minimization hypothesis as proposed by Brown (2005): higher levels of preventive nutrition at T1 were associated with a decrease in risk perception between T1 and T2 (Model 1) and with lower risk perception at T2 (Model 2). For both path models, the pattern of results indicates that people who adopt precautions were more likely to subsequently lower their risk perception at $\mathrm{T} 2$. Thus, this effect might rather indicate a time-lagged adaptation effect in the behavior-risk perception relation than a habituation effect. However, this is rather speculative and awaits further more finegraded research including more measurement points in time.

The theoretical and practical implications of adaptive accuracy and risk reappraisal effects might be considerable. Since the reduction of personal risk is a key motive for changing risk behaviors (Weinstein, 2003), decreases in perceived risk after changing behavior might be a necessary motivational prerequisite for the maintenance of protective behaviors. It could be assumed that the less people feel at risk after changing behaviors, the more they perceive the efforts invested worthwhile and the more they will be motivated to maintain the changes. This is consistent with negative reinforcement from operant theory
(Skinner, 1953). Such feedback loops of risk perceptions, however, are not explicitly included in most current health behavior models. An exception is the parallel process model, which assumes that after adopting preventive behavior, reappraisal processes are initiated (Leventhal et al., 2003; Witte, 1994).

Assuming that decreases in risk perceptions affect the motivation to maintain behavior changes, the question arises: How do people gauge the risk they face? These results suggest that there is a high correspondence between perceived risk and behavior, but a considerable variability in how behavior changes are translated into changes in perceived risk. Current theories of health behavior assume that cognitive appraisals and expectancies, such as perceived likelihood and perceived severity, are the primary determinants of risk perceptions (Renner \& Schwarzer, 2003; Weinstein, 2003). From a broader perspective, however, the process of risk perception formation could also be conceptualized from a dual processing perceptive: dual processing models distinguish between a "hot" emotional system that is simple, effortless, fast, and automatic and a "cold" deliberative system that is complex, slow, and, effortful (Loewenstein et al., 2001; Slovic et al., 2002). Thus, a dual processing perspective suggests that the variability in adaptive accuracy of risk perception is determined both by deliberate and effortful cognitive processes as well as by automatic affective reactions. Accordingly, people might have evaluated the risks emerging from BSE and FMD similarly but they may greatly differ in their emotional reactions toward risks or their "feelings of risk" (cf. Loewenstein et al., 2001), which leads to a considerable variability in adaptive accuracy in risk perceptions. This line of reasoning, in conjunction with the present findings, suggests employing multimodal measurement approaches (Lang et al., 1997) in order to fully understand the processing of risk information and the formation of risk perceptions.

\subsection{Limitations and Implications}

Apart from limitations that arise from the use of self-report measures of behavior and a nonrepresentative sample due to the assessment strategy (Internet questionnaires), the study has clear limitations with regard to the examination of risk perceptionbehavior relations. First, although the research was conducted in temporal proximity to the incidence of BSE and FMD in Germany, thus trying to capture as many participants as possible before they have changed their health behavior, it cannot be excluded 
that participants may have changed behavior before the survey. Thus, the theoretically ideal measurement point (after the occurrence of diseases and before behavioral reactions) might have been missed (cf. Weinstein et al., 1998). Additionally, the increase in preventive nutrition as well as the decrease in risk perception may have partly been generated by a response bias caused by the repeated measurement, by a regression to the mean effect, or a third variable not assessed. The measures used to assess risk perceptions refer to relative risk perception rather than absolute risk perceptions. However, in previous research, both measures for risk perception were highly positive correlated (e.g., Klar \& Giladi, 1999) and found predictive for health behavior. Additionally, individual food preferences not controlled for in this study might bias the interpretation of the data, although these individual preferences are not likely to have changed during the study period.

Despite these limitations, this study has some important implications. The results demonstrate the need for longitudinal research designs with multiple measurement points when examining risk perceptionhealth behavior relations. Weinstein et al. (1998) provide a promising framework for cross-lagged research with multiple measurement points, which should be enhanced by interventions designed to change and to examine risk perceptions and behavior changes. Moreover, according to dual processing notions, risk perceptions and changes in feelings of risk might compromise cognitive and affective facets. Thus, further risk research could be meaningfully extended by relating both cognitive and affective facets in a multimodal measurement, which assesses responses in different reactive systems (expressive and evaluative language, physiological changes, and behavior orientation; Lang et al., 1997). Finally, from a health education perspective these results suggest that successful and sustainable behavior intervention programs should not only provide information and strengthen self-regulatory skills but might also need to facilitate adaptive accuracy in risk estimates and behavioral outcomes.

\section{REFERENCES}

Armitage, C. J., \& Conner, M. (2000). Social cognition models and health behaviour: A structured review. Psychology and Health, $15,173-189$.

Ayanian, J. Z., \& Cleary, P. D. (1999). Perceived risks of heart disease and cancer among cigarette smokers. Journal of the American Medical Association, 281, 1019-1021.

Brewer, N. T., Weinstein, N. D., Cuite, C. L., \& Herrington, J. E., Jr. (2004). Risk perceptions and their relation to risk behavior. Annals of Behavioral Medicine, 27, 125-130.
Brown, S. L. (2005). Relationships between risk-taking behaviour and subsequent risk perceptions. British Journal of Psychology, $96,155-164$.

Croyle, R. T., Sun, Y. C., \& Hart, M. (1997). Processing risk factor information: Defensive biases in health-related cognitions. In K. Petrie, J. Weinman, \& J. A. Amsterdam (Eds.), Perceptions of Health and Illness: Current Research and Applications (pp. 267-290). Amsterdam: Harwood Academic Publishers.

Enders, C. K. (2001). A primer on maximum likelihood algorithms available for use with missing data. Structural Equation Modeling, 8, 128-141.

French, D. P., \& Marteau, T. (2007). Communicating risk. In S. Ayers, A. Baum, C. McManus, S. Newman, K. A. Wallston, J. Weinman, \& R. West (Eds.), Cambridge Handbook of Psychology, Health and Medicine, 2nd ed. (pp. 431-435). Cambridge: Cambridge University Press.

Gerrard, M., Gibbons, F. X., \& Bushman, B. J. (1996). Relation between perceived vulnerability to HIV and precautionary sexual behavior. Psychological Bulletin, 119, 390-409.

Gerrard, M., Gibbons, F. X., Reis Bergan, M., \& Russell, D. W. (2000). Self-esteem, self-serving cognitions, and health risk behavior. Journal of Personality, 68, 1177-1201.

Harrison, J. A., Mullen, P. D., \& Green, L. W. (1992). A metaanalysis of studies of the health belief model with adults. Health Education Research, 7, 107-116.

Johnson, E. J., \& Tversky, A. (1983). Affect, generalization, and the perception of risk. Journal of Personality and Social Psychology, 45, 20-31.

Klar, Y., \& Giladi, E. E. (1999). Are most people happier than their peers, or are they just happy? Personality and Social Psychology Bulletin, 25, 585-594.

Kunda, Z. (1990). The case for motivated reasoning. Psychological Bulletin, 108, 480-498.

Lang, P. J., Bradley, M. M., \& Cuthbert, B. N. (1997). Motivated attention: Affect, activation, and action. In P. J. Lang, R. F. Simons, \& M. Balaban (Eds.), Attention and Orienting: Sensory and Motivational Processes (pp. 97-135). Mahwah, NJ: Lawrence Erlbaum Associates.

Leventhal, H., Brissette, I., \& Leventhal, E. A. (2003). The common-sense model of self-regulation of health and illness. In L. D. Cameron \& H. Leventhal (Eds.), The Self-Regulation of Health and Illness Behavior (pp. 42-65). Howard: Routledge.

Lima, M. L. (2004). On the influence of risk perception on mental health: Living near an incinerator. Journal of Environmental Psychology, 24, 71-84.

Loewenstein, G. F., Weber, E. U., Hsee, C. K., \& Welch, N. (2001). Risk as feelings. Psychological Bulletin, 127, 267-286.

Pfister, H. R., \& Böhm, G. (2001). BSE-Sozialpsychologische Aspekte eines umstrittenen Risikos. Zeitschrift für Sozialpsychologie, 32, 213-221.

Reisen, C. A., \& Poppen, P. J. (1999). Partner-specific risk perception: A new conceptualization of perceived vulnerability to STDs. Journal of Applied Social Psychology, 29, 667-684.

Renner, B., \& Schwarzer, R. (2003). Social-cognitive factors in health behavior change. In J. Suls \& K. A. Wallston (Eds.), Social Psychological Foundations of Health and Illness (pp. 169-196). Oxford: Blackwell.

Rutter, D., \& Quine, L. (2002). Social Cognition Models and Changing Health Behaviours. Buckingham: Open University Press.

Sheeran, P., \& Abraham, C. (1996). The health belief model. In M. Conner \& P. Norman (Eds.), Predicting Health Behaviour (pp. 23-61). Buckingham: Open University Press.

Sjöberg, L., Holm, L. E., Ullén, H., \& Brandberg, Y. (2004). Tanning and risk perception in adolescents. Health, Risk \& Society, 6, 81-94.

Skinner, B. F. (1953). Science and Human Behavior. New York: Macmillan.

Slovic, P., Finucane, M., Peters, E., \& MacGregor, D. G. (2002). The affect heuristic. In T. Gilovich, D. Griffin, \& D. Kahneman 
(Eds.), Heuristics and Biases: The Psychology of Intuitive Judgment (pp. 397-420). New York: Cambridge University Press.

Slovic, P., \& Lichtenstein, S. (1982). Why study risk perceptions? Risk Analysis, 2, 83-93.

Sniehotta, F. F., Luszczynska, A., Scholz, U., \& Lippke, S. (2005). Discontinuity patterns in stages of the precaution adoption process model: Meat consumption during a livestock epidemic. British Journal of Health Psychology, 10, 221-235.

Van Der Pligt, J. (1998). Perceived risk and vulnerability as predictors of precautionary behaviour. British Journal of Health Psychology, 3, 1-14.

Weinstein, N. D. (2003). Exploring the links between risk perceptions and preventive health behavior. In J. Suls \& K. A. Wallston (Eds.), Social Psychological Foundations of Health and Illness (pp. 22-53). Malden, MA: Blackwell Publishers.
Weinstein, N. D. (2007). Misleading tests of health behavior theories. Annals of Behavioral Medicine, 33, 1-10.

Weinstein, N. D., \& Nicolich, M. (1993). Correct and incorrect interpretations of correlations between risk perceptions and risk behaviors. Health Psychology, 12, 235-245.

Weinstein, N. D., Rothman, A. J., \& Nicolich, M. (1998). Use of correlational data to examine the effects of risk perceptions on precautionary behavior. Psychology \& Health, 13, 479501.

Weyman, A. K., \& Clarke, D. D. (2003). Investigating the influence of organizational role on perceptions of risk in deep coal mines. Journal of Applied Psychology, 88, 404-412.

Witte, K. (1994). Fear control and danger control: A test of the extended parallel process model (EPPM). Communication Monographs, 61, 113-134. 
Copyright of Risk Analysis: An International Journal is the property of Blackwell Publishing Limited and its content may not be copied or emailed to multiple sites or posted to a listserv without the copyright holder's express written permission. However, users may print, download, or email articles for individual use. 